\title{
Impaired kidney function is associated with lower quality of life among community- dwelling older adults
}

\section{The screening for CKD among older people across Europe (SCOPE) study}

\author{
Rada Artzi-Medvedik ${ }^{1,2}$, Robert Kob ${ }^{3}$, Paolo Fabbietti ${ }^{4,5^{*}}$ (D), Fabrizia Lattanzio ${ }^{4}$, Andrea Corsonello ${ }^{4}$, \\ Yehudit Melzer ${ }^{2,6}$, Regina Roller-Wirnsberger ${ }^{7}$, Gerhard Wirnsberger ${ }^{8}$, Francesco Mattace-Raso ${ }^{9}$, Lisanne Tap ${ }^{9}$, \\ Pedro Gil ${ }^{10}$, Sara Lainez Martinez ${ }^{10}$, Francesc Formiga ${ }^{11}$, Rafael Moreno-González ${ }^{11}$, Tomasz Kostka ${ }^{12}$, \\ Agnieszka Guligowska ${ }^{12}$, Johan Ärnlöv ${ }^{13,14,15}$, Axel C. Carlsson ${ }^{13,14,15}$, Ellen Freiberger ${ }^{3^{*}}$, Itshak Melzer ${ }^{2^{*}}$ and on \\ behalf of the SCOPE investigators
}

\begin{abstract}
Background: Quality of life (QoL) refers to the physical, psychological, social and medical aspects of life that are influenced by health status and function. The purpose of this study was to measure the self-perceived health status among the elderly population across Europe in different stages of Chronic Kidney Disease (CKD).

Methods: Our series consisted of 2255 community-dwelling older adults enrolled in the Screening for Chronic Kidney Disease (CKD) among Older People across Europe (SCOPE) study. All patients underwent a comprehensive geriatric assessment (CGA), including included demographics, clinical and physical assessment, number of medications taken, family arrangement, Geriatric Depression Scale (GDS), Cumulative Illness Rating Scale, History of falls, Lower urinary tract symptoms, and Short Physical Performance Battery (SPPB). Estimated glomerular filtration rate (eGFR) was calculated by Berlin Initiative Study (BIS) equation. Quality of life was assessed by Euro Qol questionnaire (Euro-Qol 5D) and EQ-Visual Analogue Scale (EQ-VAS). The association between CKD (eGFR $<60,<45 \mathrm{ml}$ or $<30 \mathrm{ml} / \mathrm{min} / 1.73 \mathrm{~m}^{2}$ ) and low EQoL-VAS was investigated by multivariable logistic regression models.

(Continued on next page)
\end{abstract}

\footnotetext{
*Correspondence: p.fabbietti@inrca.it; Ellen.Freiberger@fau.de; itzikm@bgu.ac.il

${ }^{4}$ Italian National Research Center on Aging (IRCCS INRCA), Ancona, Fermo and Cosenza, Italy

${ }^{3}$ Department of Internal Medicine-Geriatrics, Institute for Biomedicine of Aging, Krankenhaus Barmherzige Brüder, Friedrich-Alexander Universität Erlangen-Nürnberg, Koberger Strasse 60, 90408 Nuremberg, Germany ${ }^{2}$ Department of Physical Therapy, Recanati School for Community Health Professions at the faculty of Health Sciences, Ben-Gurion University of the Negev, Beer-sheva, Israel

Full list of author information is available at the end of the article
}

(c) The Author(s). 2020 Open Access This article is licensed under a Creative Commons Attribution 4.0 International License, which permits use, sharing, adaptation, distribution and reproduction in any medium or format, as long as you give appropriate credit to the original author(s) and the source, provide a link to the Creative Commons licence, and indicate if changes were made. The images or other third party material in this article are included in the article's Creative Commons licence, unless indicated otherwise in a credit line to the material. If material is not included in the article's Creative Commons licence and your intended use is not permitted by statutory regulation or exceeds the permitted use, you will need to obtain permission directly from the copyright holder. To view a copy of this licence, visit http://creativecommons.org/licenses/by/4.0/ The Creative Commons Public Domain Dedication waiver (http://creativecommons.org/publicdomain/zero/1.0/) applies to the data made available in this article, unless otherwise stated in a credit line to the data. 


\begin{abstract}
(Continued from previous page)
Results: CKD was found to be significantly associated with low EQoL-VAS in crude analysis (OR $=1.47,95 \% \mathrm{Cl}=1.16$ 1.85 for eGFR<60; $\mathrm{OR}=1.38,95 \% \mathrm{Cl}=1.08-1.77$ for $\mathrm{eGFR}<45 ; \mathrm{OR}=1.57,95 \% \mathrm{Cl}=1.01-2.44$ ). Such association was no longer significant only when adjusting for SPPB $(\mathrm{OR}=1.20,95 \% \mathrm{Cl}=0.93-1.56$ for $\mathrm{eGFR}<60$; OR $=0.87,95 \% \mathrm{Cl}=0.64-$ 1.18 for $\mathrm{eGFR}<45$; $\mathrm{OR}=0.84,95 \% \mathrm{Cl}=0.50-1.42)$, $\mathrm{CIRS}$ and polypharmacy $(\mathrm{OR}=1.16,95 \% \mathrm{Cl}=0.90-1.50$ for eGFR< 60; $\mathrm{OR}=0.86,95 \% \mathrm{Cl}=0.64-1.16$ for $\mathrm{eGFR}<45 ; \mathrm{OR}=1.11,95 \% \mathrm{Cl}=0.69-1.80)$ or diabetes, hypertension and chronic obstructive pulmonary disease $(\mathrm{OR}=1.28,95 \% \mathrm{Cl}=0.99-1.64$ for $\mathrm{eGFR}<60$; $\mathrm{OR}=1.16,95 \% \mathrm{Cl}=0.88-1.52$ for $\mathrm{eGFR}<45$; $\mathrm{OR}=1.47,95 \% \mathrm{Cl}=0.92-2.34)$. The association between CKD and low EQoL-VAS was confirmed in all remaining multivariable models.
\end{abstract}

Conclusions: CKD may significantly affect QoL in community-dwelling older adults. Physical performance, polypharmacy, diabetes, hypertension and COPD may affect such association, which suggests that the impact of CKD on QoL is likely multifactorial and partly mediated by co-occurrent conditions/risk factors.

Keywords: Quality of life, Chronic kidney disease, Old adults

\section{Background}

The importance of Quality of life (QoL) in old age was acknowledged in the WHO report on healthy aging 2015 [1]. However, rising life expectancy worldwide is not limited to the healthy population, but also affects subpopulations with a history of disease, which contribute to make QoL a relevant outcome in terms of public health among older people [2].

QoL is basically a subjective condition that expresses how people are satisfied with their life and the degree of wellbeing and happiness they feel [3]. Health-related QoL refers to the physical, psychological, social, spiritual aspects of QoL that are influenced by health and healthrelated events such as diseases and their treatments $[4,5]$.

Chronic kidney disease (CKD) is among chronic diseases significantly affecting QoL among older people. Besides exerting a major effect on global health, either as a risk factor for morbidity and mortality or by causing cardiovascular disease [6], the burden of CKD in older people is also related to its complications, including impaired physical function $[7,8]$, frailty $[9,10]$, cognitive impairment [11], vision impairment [12], malnutrition [13], and sarcopenia [14]. All the above may influence QoL of older adults.

Most studies showed that severe CKD and dialysis have negative impact on Health Related QoL $[15,16]$. However, due to the slow and unpredictable nature of CKD trajectories, earlier CKD stages (e.g. stage 3a and 3b) may also significantly affect Health Related QoL [17]. The few studies investigating the impact of early stages of CKD on QoL show that QoL may be poorer than that of the general population, but better than for CKD patients on dialysis [18, 19]. In a recent systematic review, Yapa et al. [20] showed that health-related QoL may worsen when CKD symptoms (e.g. fatigue, exhaustion and drowsiness) appear.

The objective of this cross-sectional study was to investigate the QoL among older adults across Europe in early stages of $C K D$, in order to identify factors potentially influencing the relationship between kidney function and QoL.

\section{Methods}

\section{Study design and participants}

The SCOPE study (European Grant Agreement no. 436849), is a multicenter prospective cohort study involving patients older than 75 years attending geriatric and nephrology outpatient services in participating institutions in Austria, Germany, Israel, Italy, the Netherlands, Poland and Spain. Only people aged 75 or more were asked to participate because of the high prevalence of CKD in this population [21, 22]. Methods of the SCOPE study have been extensively described elsewhere [23]. Briefly, all patients attending the outpatient services at participating centers from August 2016 to August 2018 were asked to participate. Only patients signing a written informed consent entered the study. Age greater or equal to 75 years was the only inclusion criteria, the exclusion criteria were: end-stage renal disease or dialysis at time of enrollment; history of solid organ or bone marrow transplantation; active malignancy within 24 months prior to screening or metastatic cancer; life expectancy less than 6 months (based on the judgment of the study physician after careful medical history collection and diagnoses emerging from examination of clinical documentation exhibited); severe cognitive impairment (Mini Mental State Examination < 10); any medical or other reason (e.g. known or suspected patients' inability to comply with the protocol procedure) in the judgement of the investigators, that the patient was unsuitable for the study; unwilling to provide consent and limited possibility to attend followup visits. Enrolled patients underwent an extensive assessment including: demographic data, socioeconomic status, physical examination, comprehensive geriatric assessment, bioimpedance analysis, diagnoses (clinical 
history and assessment of clinical documentation exhibited by patients and/or caregivers), quality of life, physical performance, overall comorbidity and blood and urine sampling. Patients were followed-up for 24-months as previously described [23]. The study protocol was approved by ethics committees at all participating institutions, and complies with the Declaration of Helsinki and Good Clinical Practice Guidelines. The study was registered at ClinicalTrials.gov (NCT02691546). Only baseline data was used in the present study.

Overall, 2461 patients were initially enrolled in the study; 206 patients were excluded because of incomplete baseline data, thus leaving a final sample of 2255 participants to be included in the present analysis.

\section{Study variables}

QoL was assessed by EQ-Visual Analogue Scale (EQoLVAS), that is part of the Euro-Quality of Life 5D (EuroQol 5D) [24-26]. The EQ-VAS asks participants to indicate their overall health on a vertical visual analogue scale, ranging from 0 "worst possible" to 100 "best possible" health. The Euro-Qol 5D is a standardized instrument for measuring generic health rated QoL measure with one question on five different dimensions that include mobility, self-care, usual activities, pain/discomfort, and anxiety/depression. The answers given to EuroQoL 5D are scored from 1 "I have no problems ... " for perfect health to 5 "I am unable to .... " for bad health status. The 5-digit numbers for the five dimensions are combined and describe the patient's health state. The Euro-Qol 5D and EQoL-VAS was formerly validated in several different settings and clinical conditions [27-30].

Estimated glomerular filtration rate (eGFR) was calculated by Berlin Initiative Study (BIS) equation [31], and categorized as $<60,<45$ or $<30 \mathrm{ml} / \mathrm{min} / 1.73 \mathrm{~m}^{2}$.

Other variables included in the present study were: demographics, body mass index (BMI), number of diseases and medications taken, family arrangements; Basic (ADL) and Instrumental Activities of Daily Living (IADL) [32, 33]; Mini Mental State Exam (MMSE) [34]; 15-items Geriatric Depression Scale, GDS [35]; Cumulative Illness Rating Scale, CIRS [36]; History of falls; Lower urinary tract symptoms, LUTS [37]; hand grip strength [38]; Short Physical Performance Battery, SPPB [39]. Selected diagnoses, including diabetes, hypertension, stroke, hip fractures, chronic obstructive pulmonary disease, osteoporosis, Parkinson's disease and anemia were also considered as potential confounders.

\section{Statistical analysis}

Descriptive analyses of patients grouped according to EQoL-VAS (low EQoL-VAS, 0-50, intermediate EQoLVAS, 51-75, and high EQoL-VAS, 75-100) were presented. The chi-square test was used for categorical variables and ANOVA one-way test for continuous ones. Post-hoc analysis for multiple comparisons was carried out by Bonferroni correction for continuous variables and by Dunn's test for categorical ones. Therefore, multivariable logistic regression models were built to investigate the association between CKD (eGFR $<60,<45$ or $<$ $30 \mathrm{ml} / \mathrm{min} / 1.73 \mathrm{~m}^{2}$ ) and low EQoL-VAS. Logistic regression models were as follows: crude (model 1), adjusted for age and gender (model 2), furtherly adjusted by adding family arrangement (i.e., being widow) (model 3), SPPB (model 4), falls (model 5); mood status i.e., GDS > 5 (model 6), Cumulative Illness Rating Score and number of medications $\geq 5$ (model 7), LUTS (model 8), comorbidities (models 9 and 10), and anemia (model 11). All statistical analyses were performed with SPSS statistical software package version 24 (SPSS Inc., Chicago, IL, USA). Statistical significance was set at $P<0.05$.

\section{Results}

Table 1 shows that 984 out of 2255 participants (43.6\%) reported high EQoL-VAS, and 487 (21.6\%) reported low EQoL-VAS. More than half (55.96\%) were married or lived with a partner, $33.7 \%$ were widowed, $5.4 \%$ were single and $24.4 \%$ lived alone. Older adults with low EQoL-VAS (0-50) were more frequently women, single, and widowed, and had lower education compared to those with intermediate and with high EQoL-VAS (Table 1).

Table 2 shows that eGFR was lower and the prevalence of CKD was higher among patients with low EQoL-VAS, whatever was the eGFR threshold used. Polypharmacy was also highly prevalent among patients with low EQoL-VAS, who also exhibited higher average CIRS score, greater prevalence of (LUTS) and comorbidities and lower hemoglobin values (Table 2).

In regard to physical and emotional status, SPPB scores and hand grip strength were lower and the prevalence of $\mathrm{ADL} / \mathrm{IADL}$ dependency depression, cognitive impairment and history of falls was higher (Table 3).

In logistic regression analyses (Table 4), CKD was significantly associated with the outcome independent of the eGFR threshold considered in the analysis. After adjusting for age, sex, being widowed, history of falls, GDS > 5, LUTS, stroke, hip fracture, Parkinson's disease, and anemia the association between eGFR and low EQoL-VAS remained substantially unchanged (Table 4). When we adjusted for SPPB (model 4), the association between eGFR and the outcome was no longer significant. Indeed, SPPB score qualified as a significant negative correlate of low EQoL-VAS (OR $=0.72 ; 95 \% \mathrm{CI}=$ $0.69-0.76$ in the eGFR $<60$ analysis, $\mathrm{OR}=0.72 ; 95 \% \mathrm{CI}=$ $0.68-0.75$ in the GFR $<45$ analysis and $\mathrm{OR}=0.72$; $95 \% \mathrm{CI}=0.68-0.75$ in the GFR $<30$ analysis). Similarly, CIRS $(\mathrm{OR}=1.10 ; 95 \% \mathrm{CI}=1.07-1.13$ in the eGFR $<60$ 
Table 1 Sociodemographic characteristics, according to EQOL-VAS category

\begin{tabular}{|c|c|c|c|c|c|c|}
\hline Variable & $\begin{array}{l}\text { All participants } \\
(\boldsymbol{n}=2255)\end{array}$ & $\begin{array}{l}\text { Group A Low EQoL- } \\
\text { VAS 0-50 }(\boldsymbol{n}=487)\end{array}$ & $\begin{array}{l}\text { Group B Intermediate EQoL- } \\
\text { VAS 51-75 }(\boldsymbol{n}=784)\end{array}$ & $\begin{array}{l}\text { Group C High EQoL-VAS } \\
76-100(\boldsymbol{n}=984)\end{array}$ & $p$-value & post hoc \\
\hline \multirow[t]{2}{*}{$\begin{array}{l}\text { Euro QoL questionnaire } \\
\text { (Euro-Qol 5D) }\end{array}$} & $7.0(4.0)$ & $10.0(5.0)$ & $8.0(4.0)$ & $6.0(3.0)$ & $<0.001$ & $\begin{array}{l}a \text { vs. } b \\
a \text { vs. } c\end{array}$ \\
\hline & & & & & & $b$ vs. $c$ \\
\hline Age (years) & $79.5(5.9)$ & $80.0(6.4)$ & $79.5(6.0)$ & $79.2(5.3)$ & 0.009 & $a$ vs. $c$ \\
\hline \multirow[t]{3}{*}{ Sex: female } & $1255(55.7)$ & $332(68.2)$ & $423(54.0)$ & $500(50.8)$ & $<0.001$ & $a$ vs. $b$ \\
\hline & & & & & & $a$ vs. c \\
\hline & & & & & & $b$ vs. $c$ \\
\hline \multirow[t]{2}{*}{ Body mass index, BMI (kg/m²) } & $27.3(5.7)$ & $28.3(6.1)$ & $27.6(5.9)$ & $26.7(5.3)$ & $<0.001$ & a vs.c \\
\hline & & & & & & $b$ vs. $c$ \\
\hline \multicolumn{7}{|l|}{ Marital status } \\
\hline Single & $121(5.4)$ & $35(7.2)$ & $40(5.1)$ & $46(4.7)$ & $<0.001$ & $a$ vs. $b$ \\
\hline $\begin{array}{l}\text { Married/ living with a } \\
\text { partner }\end{array}$ & $1253(55.6)$ & $227(46.6)$ & $439(56.0)$ & $587(59.7)$ & & $a$ vs. $c$ \\
\hline Separated/divorced & $120(5.3)$ & $31(6.4)$ & $47(6.0)$ & $42(4.3)$ & & \\
\hline Widowed & $761(33.7)$ & $194(39.8)$ & $258(32.9)$ & $309(31.4)$ & & \\
\hline \multirow[t]{2}{*}{ Education (years) } & $11.0(7.0)$ & $10.0(5.0)$ & $12.0(7.0)$ & $12.0(7.0)$ & $<0.001$ & $a$ vs. $b$ \\
\hline & & & & & & $a$ Vs.c \\
\hline
\end{tabular}

analysis, $\mathrm{OR}=1.11 ; 95 \% \mathrm{CI}=1.01-1.14$ in the $\mathrm{GFR}<45$ analysis and $\mathrm{OR}=1.10 ; 95 \% \mathrm{CI}=1.07-1.14$ in the $\mathrm{GFR}<$ 30 analysis) and number of medications $(\mathrm{OR}=1.98$; $95 \% \mathrm{CI}=1.49-2.62$ for $\mathrm{eGFR}<60$ analysis, $\mathrm{OR}=2.01$; $95 \% \mathrm{CI}=1.51-2.66$ in the GFR $<45$ analysis and $\mathrm{OR}=$ $1.99 ; 95 \% \mathrm{CI}=1.50-2.64$ respectively in the $\mathrm{GFR}<30$ analysis) were significantly associated with the study outcome in model 7. Diabetes $(\mathrm{OR}=1.42 ; 95 \% \mathrm{CI}=1.09$ 1.85 in $\mathrm{eGFR}<60$ analysis, $\mathrm{OR}=1.40 ; 95 \% \mathrm{CI}=1.07-1.84$ in $\mathrm{eGFR}<45$ analysis and $\mathrm{OR}=1.42 ; 95 \% \mathrm{CI}=1.09-1.86$ in $\mathrm{eGFR}<30$ analysis), hypertension $(\mathrm{OR}=1.83 ; 95 \% \mathrm{CI}=$ $1.36-2.45$ in eGFR $<60$ analysis, $\mathrm{OR}=1.87 ; 95 \% \mathrm{CI}=$ $1.40-2.51$ in eGFR $<45$ analysis and $\mathrm{OR}=1.86 ; 95 \% \mathrm{CI}=$ 1.39-2.49 in eGFR<30 analysis), and chronic obstructive pulmonary disease $(\mathrm{OR}=1.99 ; 95 \% \mathrm{CI}=1.41-2.82$ in eGFR $<60$ analysis, $\mathrm{OR}=2.01 ; 95 \% \mathrm{CI}=1.43-2.84$ in $\mathrm{eGFR}<45$ analysis and $\mathrm{OR}=2.03 ; 95 \% \mathrm{CI}=1.44-2.87$ in eGFR $<30$ analysis) also qualified as significant correlates of low EQoL-VAS in model 9.

\section{Discussion}

The main finding of the present study is the association between CKD and EQoL-VAS among older communitydwelling people free from end-stage renal disease. Interestingly, such association was confirmed with all eGFR thresholds used (namely, stages 3a, 3b and 4). Thus, our results add to the present knowledge by demonstrating that early stages of CKD may significantly affect QoL among older people.
Former studies clearly showed that end-stage renal disease and dialysis are associated with low QoL [15, 16, $40,41]$, and few studies reported that even early stages of CKD may significantly affect QoL [17-20]. Our findings are clearly different from that reported in a recent cross-sectional analysis of the Irish Longitudinal Study on Ageing showing that creatinine-based eGFR may contribute little to QoL [42]. However, difference in age of the enrolled populations likely account for this apparent discrepancy. Indeed, only people aged 75 or more were enrolled in the present study, while people enrolled in the Irish study were younger (median age 61 years, interquartile range 55-68) [42]. Thus, in the light of results from the present study and the above evidence, the need of a patient-centered approach including universal outcomes to CKD care among older people [43] could be further suggested.

Main mechanisms linking CKD to QoL among older people are likely linked to the complex profile of older patients with CKD, which is known to be characterized by impaired physical function $[7,8]$, frailty $[9,10]$, cognitive impairment [11], vision impairment [12], malnutrition [13], and sarcopenia [14]. The finding that selected variable, such as physical performance, comorbidity and polypharmacy, may significantly affect the relationship between CKD and QoL is in keeping with such interpretation. Former studies showed that reduced renal function may be associated with poorer physical performance in older patients [7], and impaired SPPB contributes to describe the profile of older CKD patients 
Table 2 Clinical (Medical conditions) and laboratory parameters according to the EQoL-VAS category presented as N (\%)

\begin{tabular}{|c|c|c|c|c|c|c|}
\hline Variable & $\begin{array}{l}\text { All participants } \\
(\mathrm{n}=2255)\end{array}$ & $\begin{array}{l}\text { Group A Low EQoL-VAS } \\
(0-50)(n=487)\end{array}$ & $\begin{array}{l}\text { Group B Intermediate EQoL- } \\
\text { VAS }(51-75)(n=784)\end{array}$ & $\begin{array}{l}\text { Group C High EQoL-VAS } \\
(76-100)(n=984)\end{array}$ & $p$-value & post hoc \\
\hline eGFR, $\mathrm{ml} / \mathrm{min} / 1.73 \mathrm{~m}^{2}$ & $54.2(19.6)$ & $53.2(19.7)$ & $54.1(21.2)$ & $55.7(18.4)$ & 0.005 & a vs.c \\
\hline \multirow[t]{3}{*}{$<60$} & $1423(63.1)$ & $336(69.0)$ & $494(63.0)$ & $593(60.3)$ & 0.005 & $a$ vs. $b$ \\
\hline & & & & & & $a$ vs. $c$ \\
\hline & & & & & & $b$ vs. $c$ \\
\hline \multirow[t]{2}{*}{$<45$} & $560(24.8)$ & $137(28.1)$ & $206(26.3)$ & $217(22.1)$ & 0.020 & $a$ vs. $c$ \\
\hline & & & & & & $b$ vs. $c$ \\
\hline$<30$ & $141(6.3)$ & $37(7.6)$ & $55(7.0)$ & $49(5.0)$ & 0.082 & \\
\hline \multirow[t]{3}{*}{ Diabetes } & $568(25.2)$ & $137(28.1)$ & $225(28.7)$ & $206(21)$ & $<0.001$ & $a$ vs. $b$ \\
\hline & & & & & & $a$ vs. $c$ \\
\hline & & & & & & $b$ vs. $c$ \\
\hline \multirow[t]{2}{*}{ Hypertension } & $1732(76.8)$ & $409(84.0)$ & $625(79.7)$ & $698(70.9)$ & $<0.001$ & $a$ vs. $b$ \\
\hline & & & & & & $\begin{array}{l}a \text { vs. } c b \\
\text { vs.c }\end{array}$ \\
\hline \multirow[t]{3}{*}{ Stroke } & $131(5.8)$ & $44(9.0)$ & $50(6.4)$ & $37(3.8)$ & $<0.001$ & $a$ vs. $b$ \\
\hline & & & & & & $a$ vs. $c b$ \\
\hline & & & & & & vs. C \\
\hline \multirow[t]{2}{*}{ Hip Fractures } & $111(4.9)$ & $36(7.4)$ & $44(5.6)$ & $31(3.2)$ & 0.001 & $a$ vs. $b$ \\
\hline & & & & & & $\begin{array}{l}a \text { vs. c } b \\
\text { vs.c }\end{array}$ \\
\hline \multirow{3}{*}{$\begin{array}{l}\text { Chronic obstructive } \\
\text { pulmonary disease (COPD) }\end{array}$} & $267(11.8)$ & $76(15.6)$ & 99 (12.6) & $92(9.3)$ & 0.002 & $a$ vs. $b$ \\
\hline & & & & & & $a$ vs. $c$ \\
\hline & & & & & & $b$ vs. $c$ \\
\hline \multirow[t]{3}{*}{ Osteoporosis } & $688(30.5)$ & $186(38.2)$ & $252(32.1)$ & $250(25.4)$ & $<0.001$ & $a$ vs. $b$ \\
\hline & & & & & & $a$ vs. $c$ \\
\hline & & & & & & $b$ vs. $c$ \\
\hline \multirow[t]{2}{*}{ Parkinson's disease } & $45(2.0)$ & $22(4.5)$ & $11(1.4)$ & $12(1.2)$ & $<0.001$ & $a$ vs. $b$ \\
\hline & & & & & & $a$ vs. $c$ \\
\hline \multirow[t]{2}{*}{ Anemia } & $477(21.2)$ & $137(28.1)$ & $156(19.9)$ & $184(18.7)$ & $<0.001$ & $a$ vs. $b$ \\
\hline & & & & & & $a$ vs. $c$ \\
\hline \multirow{2}{*}{$\begin{array}{l}\text { Cumulative Illness Rating } \\
\text { Score (CIRS) }\end{array}$} & $8.0(6.0)$ & $9.0(7.0)$ & $8.0(7.0)$ & $7.0(6.0)$ & $<0.001$ & $a$ vs. $b$ \\
\hline & & & & & & $\begin{array}{l}a \text { vs. } c b \\
\text { vs. } c\end{array}$ \\
\hline \multirow{3}{*}{$\begin{array}{l}\text { Take } \geq 5 \text { current } \\
\text { medications }\end{array}$} & $1509(66.9)$ & 389 (79.9) & $554(70.7)$ & $566(57.5)$ & $<0.001$ & $a$ vs. $b$ \\
\hline & & & & & & $a$ vs. $c b$ \\
\hline & & & & & & vs. C \\
\hline \multirow{3}{*}{$\begin{array}{l}\text { Lower urinary tract } \\
\text { symptoms (LUTS) }\end{array}$} & $653(29.0)$ & $173(35.5)$ & $257(32.8)$ & $223(22.7)$ & $<0.001$ & $a$ vs. $b$ \\
\hline & & & & & & $a$ vs. $c$ \\
\hline & & & & & & $b$ vs. $c$ \\
\hline Hemoglobin $(\mathrm{Hb})$ & $13.5 \pm 1.9$ & $13.1 \pm 1.5$ & $13.5 \pm 1.4$ & $13.7 \pm 1.4$ & $<0.001$ & $a$ vs. $c$ \\
\hline
\end{tabular}

NOTE. Values are mean \pm SD for continuous normal distributions, $\mathrm{n}$ (\%) for categorical variables, and median (interquartile range) for not normal distributions

with increased risk of death [44]. Additionally, besides confirming the impact of physical performance on QoL [45], our study also showed that the average difference across QoL groups observed in our study in regards to SPPB score was clearly higher compared to minimum clinically meaningful difference (i.e. 0.5 points) [46].
These findings further sustain the need of developing exercise interventions to improve physical performance among CKD patients to counteract deterioration of QoL $[47,48]$.

Overall comorbidity (i.e. CIRS score) and selected diagnoses (i.e. diabetes, hypertension and chronic 
Table 3 Physical, cognitive and emotional status according to the EQoL-VAS category

Variable All participants Group A Low EQoL-VAS Group B Intermediat

Group C High EQoL-VAS $\boldsymbol{p}$-value post hoc

$(n=2255) \quad 0-50(n=487)$

EQoL-VAS 51-75 $(n=784) \quad 76-100(n=984)$

ADL dependent or intensive

$107(4.8)$

$50(10.3)$

$33(4.2)$

assistance $>=1$

IADL dependent or intensive

$993(44.1)$

assistance $>=1$

$993(44.1) \quad 251(51.9)$

SPPB total score (avarge \pm SD)

9 (4)

7 (6)

$9(4)$

$24(2.4)$

$<0.001$ a vs. $b$

a vs. C

$b$ vs $c$

SPPB Balance score

\begin{tabular}{|c|c|c|c|c|c|c|}
\hline $\begin{array}{l}\text { Held SBS }<10 \mathrm{~s} \text { and held } 10 \mathrm{~s} \\
\text { SBS but unable ST as severe } \\
\text { balance limitation }\end{array}$ & $432(20.1)$ & $151(34.0)$ & $148(20.0)$ & $133(13.8)$ & $<0.001$ & $a$ vs. $b$ \\
\hline $\begin{array}{l}\text { Moderate limitation Held ST for } \\
10 \text { s und held FT till } 9 \text { s. }\end{array}$ & $410(19.1)$ & $99(22.3)$ & $143(19.3)$ & $168(17.4)$ & & \\
\hline \multirow[t]{2}{*}{ No limitation: hold FT for $10 \mathrm{~s}$. } & $1307(60.8)$ & $194(43.7)$ & $450(60.7)$ & $663(68.8)$ & & $a$ vs. $c$ \\
\hline & & & & & & $b$ vs. $c$ \\
\hline \multicolumn{7}{|l|}{ SPPB gait score } \\
\hline$<4.82 \mathrm{~s}$ & $1138(50.5)$ & $157(32.2)$ & $372(47.4)$ & 609 (61.9) & $<0.001$ & $a$ vs. $b$ \\
\hline $4.82-8.7 \mathrm{~s}$ & $902(40.0)$ & $224(46.0)$ & $348(44.4)$ & $330(33.5)$ & & $a$ vs. $c$ \\
\hline$>8.70$ s and Unable & $215(9.5)$ & $106(21.8)$ & $64(8.2)$ & $45(4.6)$ & & $b$ vs. $c$ \\
\hline \multicolumn{7}{|l|}{-sit to stand score } \\
\hline$\leq 11.19 \mathrm{~s}$ & $654(32.2)$ & $62(16.5)$ & $222(31.2)$ & $370(39.2)$ & $<0.001$ & $a$ vs. $b$ \\
\hline $11.20-16.69 s$ & $924(45.5)$ & $163(43.4)$ & $318(44.7)$ & $443(46.9)$ & & $a$ Vs. $c$ \\
\hline$>16.70$ s and $>60$ s or unable & $454(22.3)$ & $151(40.2)$ & $171(24.1)$ & $132(14.0)$ & & $b$ Vs. $c$ \\
\hline \multirow[t]{3}{*}{ Hand grip strength (avarge \pm SD) } & $21.0(12.8)$ & $19.4(8.3)$ & $22.9(9.3)$ & $24.1(9.2)$ & $<0.001$ & $a$ vs. $b$ \\
\hline & & & & & & $a$ vs. $c$ \\
\hline & & & & & & $b$ vs. $c$ \\
\hline \multirow[t]{2}{*}{ GDS score (avarge \pm SD) } & $2(3)$ & $4(4)$ & $2(3)$ & $1(3)$ & $<0.001$ & $a$ vs. $b$ \\
\hline & & & & & & $a$ vs. $c$ \\
\hline \multirow[t]{3}{*}{$\mathrm{GDS}>5$} & $316(14.0)$ & $152(31.2)$ & $104(13.3)$ & $60(6.1)$ & $<0.001$ & $a$ vs. $b$ \\
\hline & & & & & & $a$ Vs. $c$ \\
\hline & & & & & & $b$ vs. $c$ \\
\hline \multirow[t]{2}{*}{ MMSE score (avarge \pm SD) } & $29(3)$ & $28(4)$ & $29(3)$ & $29(3)$ & $<0.001$ & $a$ vs. $b$ \\
\hline & & & & & & $a$ vs. $c$ \\
\hline \multirow[t]{3}{*}{ MMSE < 24} & $159(7.1)$ & $50(10.3)$ & $46(5.9)$ & $63(6.4)$ & 0.007 & $a$ vs. $b$ \\
\hline & & & & & & $a$ vs. $c$ \\
\hline & & & & & & $b$ Vs. $c$ \\
\hline \multirow[t]{3}{*}{ Fall at the past 12 months } & $746(33.1)$ & $207(42.5)$ & $267(34.1)$ & $272(27.6)$ & $<0.001$ & $a$ vs. $b$ \\
\hline & & & & & & $a$ vs. $c$ \\
\hline & & & & & & $b$ vs. $c$ \\
\hline
\end{tabular}

NOTE. Values are mean \pm SD for continuous normal distributions, $\mathrm{n}$ (\%) for categorical variables, and median (interquartile range) for not normal distributions Abbreviations: NS, not significance; ADL, activities of daily living; GDS, Geriatric Depression Scale; iADL, instrumental activities of daily living; MMSE, Mini-Mental State Examination; SPPB, Short Physical performance Battery 
Table 4 Probability of having low quality of life (QoL 0-50) in CKD groups with older adults with eGFR $<45 \mathrm{ml} / \mathrm{min} / 1.73 \mathrm{~m}^{2}$ vs. older adults with eGFR $>=45 \mathrm{ml} / \mathrm{min} / 1.73 \mathrm{~m}^{2}$ (left column), and right column CKD groups with older adults with eGFR $<30 \mathrm{ml} / \mathrm{min} / 1.73 \mathrm{~m}^{2}$ vs. $\mathrm{eGFR}>=30 \mathrm{ml} / \mathrm{min} / 1.73 \mathrm{~m}^{2}$

\begin{tabular}{|c|c|c|c|}
\hline Predictors & $\begin{array}{l}\text { OR }(95 \% \text { Cl }) \\
\text { GFR }<60\end{array}$ & $\begin{array}{l}\mathrm{OR}(95 \% \mathrm{Cl}) \\
\mathrm{GFR}<45\end{array}$ & $\begin{array}{l}\text { OR }(95 \% \mathrm{Cl}) \\
\mathrm{GFR}<30\end{array}$ \\
\hline Model 1. CKD alone & $1.47(1.16-1.85)$ & $1.38(1.08-1.77)$ & $1.57(1.01-2.44)$ \\
\hline Model 2. Model 1 adjusted for age and sex & $1.47(1.15-1.86)$ & $1.40(1.07-1.82)$ & $1.71(1.08-2.69)$ \\
\hline Model 3. Model 2 adjusted for family arrangement (widow) & $1.47(1.15-1.87)$ & $1.40(1.07-1.82)$ & $1.71(1.08-2.70)$ \\
\hline Model 4. Model 2 adjusted for SPPB total score & $1.20(0.93-1.56)$ & $0.87(0.64-1.18)$ & $0.84(0.50-1.42)$ \\
\hline Model 5. Model 2 adjusted for At least 1 fall past 12 months & $1.47(1.16-1.88)$ & $1.39(1.06-1.82)$ & $1.70(1.07-2.69)$ \\
\hline Model 6. Model 2 adjusted for GDS > 5 & $1.48(1.15-1.91)$ & $1.42(1.07-1.88)$ & $1.78(1.11-2.87)$ \\
\hline $\begin{array}{l}\text { Model 7. Model } 2 \text { adjusted for Cumulative Illness Rating Score and number of } \\
\text { medications } \geq 5\end{array}$ & $1.16(0.90-1.50)$ & $0.86(0.64-1.16)$ & $1.11(0.69-1.80)$ \\
\hline Model 8. Model 2 adjusted for lower Urinary tract symptoms & $1.50(1.18-1.92)$ & $1.47(1.12-1.92)$ & $1.93(1.22-3.07)$ \\
\hline $\begin{array}{l}\text { Model 9. Model } 2 \text { adjusted for Diabetes, Hypertension, and Chronic obstructive } \\
\text { pulmonary disease }\end{array}$ & $1.28(0.99-1.64)$ & $1.16(0.88-1.52)$ & $1.47(0.92-2.34)$ \\
\hline Model 10. Model 2 adjusted for Stroke, Hip fracture, and Parkinson's Disease & $1.48(1.16-1.89)$ & $1.42(1.08-1.87)$ & $1.65(1.03-2.62)$ \\
\hline Model 11. Model 2 adjusted for Anemia & $1.47(1.15-1.86)$ & $1.49(1.0-2.22)$ & $1.51(1.01-2.25)$ \\
\hline
\end{tabular}

Abbreviations: $\mathrm{Cl}$, confidence interval; $\mathrm{OR}$, odds ratio

obstructive pulmonary disease), are known to be major determinant of CKD or highly prevalent comorbidities among older patients with CKD [49, 50]. Diabetes, hypertension and chronic obstructive pulmonary disease were also found associated with QoL decline in dialysis patients [51-53], and our findings are consistent with the hypothesis that these comorbidities may negatively affect QoL even among older people with less severe degrees of CKD. On the other hand, diabetic patients maintaining high level of physical activity and exercise were exhibited better QoL [54]. Finally, CIRS was found associated with QoL in community-dwelling older adults [55], as was polypharmacy [56]. Thus, our findings that the addition of these variables to the multivariable models may blunt the association between CKD and QoL further strengthen their role as important correlates of QoL among older people and suggests that the impact of CKD on QoL may be at least partly mediated by risk factors typically observed among older people.

Limitations of the present study deserve to be mentioned. The cross-sectional design does not allow to derive causal relationships between CKD and QoL. However, the ongoing collection of prospective data in the context of the SCOPE study is expected to provide further insight in this topic. Additionally, we enrolled a population of relatively healthy older communitydwelling volunteer, thus prone to volunteer bias, which may reduce generalizability of the present finding to the general older population. Finally, only creatinine-based eGFR was used as a measure of kidney function in our study, and recent evidence suggests that using different biomarkers (e.g. cystatin C) may yield different results [42]. As for strength, we had the opportunity to investigate the association between CKD and QoL after adjusting for several important confounders thanks to the comprehensive assessment carried out during the study visits.

\section{Conclusions}

Our study shows that in older adults self-perceived QoL is multifactorial and influenced by medical, emotional, functional and social conditions. We observed a significant association of CKD stages 3a, 3b and 4 with QoL. Such association was confirmed after adjusting for sociodemographic and clinical factors. Efforts should be made to decrease the negative effects of potentially modifiable factors, such as physical performance, and to better manage comorbidities. Further longitudinal studies are need to clarify whether targeting patients with early stages of CKD may help to prevent QoL decline.

\section{Abbreviations}

QoL: Quality of life; CKD: Chronic Kidney Disease; EQ-VAS: EQ-Visual Analogue Scale; Euro-Qol 5D: Euro-Quality of life 5D; eGFR: Glomerular filtration rate; BIS: Berlin Initiative Study eq.; ADL: Basic Activities of Daily; IADL: Instrumental Activities of Daily Living; GDS: Geriatric Depression Scale; CIRS: Cumulative Illness Rating Scale; LUTS: Lower urinary tract symptoms; SPPB: Short Physical Performance Battery; OR: Odds Ratio

\section{Acknowledgements}

SCOPE study investigators.

Coordinating center, Fabrizia Lattanzio, Italian National Research Center on Aging (INRCA), Ancona, Italy - Principal Investigator. Andrea Corsonello, Silvia Bustacchini, Silvia Bolognini, Paola D'Ascoli, Raffaella Moresi, Giuseppina Di Stefano, Cinzia Giammarchi, Anna Rita Bonfigli, Roberta Galeazzi, Federica Lenci, Stefano Della Bella, Enrico Bordoni, Mauro Provinciali, Robertina Giacconi, Cinzia Giuli, Demetrio Postacchini, Sabrina Garasto, Annalisa Cozza, Francesco Guarasci, Sonia D'Alia - Italian National Research Center on Aging (INRCA), Ancona, Fermo and Cosenza, Italy - Coordinating staff. Romano Firmani, Moreno Nacciariti, Mirko Di Rosa, Paolo Fabbietti - Technical and statistical support. 


\section{Participating centers.}

Department of Internal Medicine, Medical University of Graz, Austria: Gerhard Hubert Wirnsberger, Regina Elisabeth Roller-Wirnsberger, Carolin Herzog, Sonja Lindner.

Section of Geriatric Medicine, Department of Internal Medicine, Erasmus MC, University Medical Center Rotterdam, The Netherlands: Francesco MattaceRaso, Lisanne Tap, Gijsbertus Ziere, Jeannette Goudzwaard. Department of Geriatrics, Healthy Ageing Research Centre, Medical University of Lodz, Poland: Tomasz Kostka, Agnieszka Guligowska, Łukasz Kroc Bartłomiej K Sołtysik, Małgorzata Pigłowska, Agnieszka Wójcik, Zuzanna Chrzastek, Natalia Sosowska, Anna Telążka, Joanna Kostka, Elizaveta Fife, Katarzyna Smyj, Kinga Zel.

The Recanati School for Community Health Professions at the faculty of Health Sciences at Ben-Gurion University of the Negev, Israel: Rada ArtziMedvedik, Yehudit Melzer, Mark Clarfield, Itshak Melzer; and Maccabi Healthcare services southern region, Israel: Rada Artzi-Medvedik, Ilan Yehoshua, Yehudit Melzer.

Geriatric Unit, Internal Medicine Department and Nephrology Department, Hospital Universitari de Bellvitge, Institut d'Investigació Biomèdica de Bellvitge - IDIBELL, L'Hospitalet de Llobregat, Barcelona, Spain: Francesc Formiga, Rafael Moreno-González, Xavier Corbella, Yurema Martínez, Carolina Polo, Josep Maria Cruzado.

Department of Geriatric Medicine, Hospital Clínico San Carlos, Madrid: Pedro Gil Gregorio, Sara Laínez Martínez, Mónica González Alonso, Jose A. Herrero Calvo, Fernando Tornero Molina, Lara Guardado Fuentes, Pamela Carrillo García, María Mombiedro Pérez.

Department of General Internal Medicine and Geriatrics, Krankenhaus Barmherzige Brüder Regensburg and Institute for Biomedicine of Aging Friedrich-Alexander-Universität Erlangen-Nürnberg, Germany: Alexandra Renz, Susanne Muck, Stephan Theobaldy, Andreas Bekmann, Revekka Kaltsa, Sabine Britting, Robert Kob, Christian Weingart, Ellen Freiberger, Cornel Sieber. Department of Medical Sciences, Uppsala University, Sweden: Johan Ärnlöv, Axel Carlsson, Tobias Feldreich.

Scientific advisory board (SAB)

Roberto Bernabei, Catholic University of Sacred Heart, Rome, Italy. Christophe Bula, University of Lausanne, Switzerland. Hermann Haller, Hannover Medical School, Hannover, Germany. Carmine Zoccali, CNR-IBIM Clinical Epidemiology and Pathophysiology of Renal Diseases and Hypertension, Reggio Calabria, Italy.

Data and Ethics Management Board (DEMB)

Dr. Kitty Jager, University of Amsterdam, The Netherlands.

Dr. Wim Van Biesen, University Hospital of Ghent, Belgium.

Paul E. Stevens, East Kent Hospitals University NHS Foundation Trust, Canterbury, United Kingdom.

We thank the BioGer IRCCS INRCA Biobank for the collection of the SCOPE samples.

\section{About this supplement}

This article has been published as part of BMC Geriatrics Volume 20 Supplement 12,020: The Screening for Chronic Kidney Disease among Older People across Europe (SCOPE) project: findings from cross-sectional analysis. The full contents of the supplement are available at https://bmcgeriatr.biomedcentral.com/articles/supplements/volume-20-supplement-1.

\section{Authors' contributions}

RA: data collection, manuscript drafting and revision. EF \& IM: participated in study protocol design, Data collection, and manuscript drafting and revision. RK: manuscript drafting and revision. YM: coordinated study protocol and data collection, participated in manuscript drafting. PF: data management and statistical analyses, manuscript drafting and revision. FL, AC: conceived the study, coordinated study protocol and data collection, participated in manuscript drafting and revision. FM, LT, JÄ, ACC, RRW, GW, TK, AG, PG, SLM, FF, RMG: participated in study protocol design, Data collection, and manuscript drafting and revision. All authors read and approved the final manuscript.

\section{Funding}

SCOPE study and publication costs are funded by the European Union Horizon 2020 program, under the Grant Agreement n 634869. Funding body had no role in the design of the study and collection, analysis, and interpretation of data and in writing the manuscript.

\section{Availability of data and materials}

Data will be available for SCOPE researchers through the project website (www.scopeproject.eu).

\section{Ethics approval and consent to participate}

The study protocol was approved by ethics committees at all participating institutions, and complies with the Declaration of Helsinki and Good Clinical Practice Guidelines. All patients signed a written informed consent to be enrolled. Only baseline data are used in the present study. Ethics approvals have been obtained by Ethics Committees in participating institutions as follows:

Italian National Research Center on Aging (INRCA), Italy, \#2015 0522 IN January 27, 2016.

University of Lodz, Poland, \#RNN/314/15/KE, November 17, 2015. Medizinische Universität Graz, Austria, \#28-314 ex 15/16, August 5, 2016. Erasmus Medical Center Rotterdam, The Netherland, \#MEC-2016-036 \#NL56039.078.15, v.4, March 7, 2016.

Hospital Clínico San Carlos, Madrid, Spain, \# 15/532-E_ BC, September 16, 2016.

Bellvitge University Hospital Barcellona, Spain, \#PR204/15, January 29, 2016. Friedrich-Alexander University Erlangen-Nürnberg, Germany, \#340_15B, January 21, 2016.

Helsinki committee in Maccabi Healthcare services, Bait Ba-lev, Bat Yam, Israel, \#45/2016, July 24, 2016.

\section{Consent for publication}

Not applicable.

\section{Competing interests}

The authors declare that they have no competing interests.

\section{Author details}

'Department of Nursing, Recanati School for Community Health Professions at the faculty of Health Sciences, Ben-Gurion University of the Negev, Beer-sheva, Israel. ${ }^{2}$ Department of Physical Therapy, Recanati School for Community Health Professions at the faculty of Health Sciences, Ben-Gurion University of the Negev, Beer-sheva, Israel. ${ }^{3}$ Department of Internal Medicine-Geriatrics, Institute for Biomedicine of Aging, Krankenhaus Barmherzige Brüder, Friedrich-Alexander Universität Erlangen-Nürnberg, Koberger Strasse 60, 90408 Nuremberg, Germany. ${ }^{4}$ Italian National Research Center on Aging (IRCCS INRCA), Ancona, Fermo and Cosenza, Italy. ${ }^{5}$ Laboratory of Geriatric Pharmacoepidemiology and Biostatistics, IRCCS INRCA, Via S. Margherita 5, 60124 Ancona, Italy. ${ }^{6}$ Maccabi Health Organization, Negev district, Tel Aviv-Yafo, Israel. ${ }^{7}$ Department of Internal Medicine, Medical University of Graz, Graz, Austria. ${ }^{8}$ Division of Nephrology, Department of Internal Medicine, Medical University of Graz, Graz, Austria. ${ }^{9}$ Department of Internal Medicine, Section of Geriatric Medicine, Erasmus MC, University Medical Center Rotterdam, Rotterdam, The Netherlands.

${ }^{10}$ Department of Geriatric Medicine, Hospital Clinico San Carlos, Madrid, Spain. " ${ }^{11}$ Geriatric Unit, Internal Medicine Department, Bellvitge University Hospital - IDIBELL - L'Hospitalet de Llobregat, Barcelona, Spain.

${ }^{12}$ Department of Geriatrics, Healthy Ageing Research Centre, Medical University of Lodz, Lodz, Poland. ${ }^{13}$ Department of Medical Sciences, Uppsala University, Uppsala, Sweden. ${ }^{14}$ School of Health and Social Studies, Dalarna University, Falun, Sweden. ${ }^{15}$ Division of Family Medicine, Department of Neurobiology, Care Sciences and Society, Karolinska Institutet, Huddinge, Sweden

Received: 6 August 2020 Accepted: 11 August 2020

Published: 2 October 2020

\section{References}

1. WHO. World report on Ageing and Health. 2015. https://www.who.int/ ageing/events/world-report-2015-launch/en/.

2. Meyer AC, Drefahl S, Ahlbom A, Lambe M, Modig K. Trends in life expectancy: did the gap between the healthy and the ill widen or close? BMC Med. 2020:18(1):41.

3. The World Health Organization Quality of Life Assessment (WHOQOL) development and general psychometric properties. Soc Sci Med. 1998. 46(12):1569-85. 
4. Ferrans CE, Zerwic JJ, Wilbur JE, Larson JL. Conceptual model of healthrelated quality of life. J Nurs Scholarsh. 2005;37(4):336-42.

5. Karimi M, Brazier J. Health, health-related quality of life, and quality of life: what is the difference? Pharmacoeconomics. 2016;34(7):645-9.

6. Collaboration GBDCKD. Global, regional, and national burden of chronic kidney disease, 1990-2017: a systematic analysis for the global burden of disease study 2017. Lancet. 2020;395(10225):709-33.

7. Lattanzio F, Corsonello A, Abbatecola AM, Volpato S, Pedone C, Pranno L, Laino I, Garasto S, Corica F, Passarino G, et al. Relationship between renal function and physical performance in elderly hospitalized patients. Rejuvenation Res. 2012;15(6):545-52.

8. Pedone C, Corsonello A, Bandinelli S, Pizzarelli F, Ferrucci L, Incalzi RA. Relationship between renal function and functional decline: role of the estimating equation. J Am Med Dir Assoc. 2012;13(1):84 e11-84.

9. Roshanravan B, Khatri M, Robinson-Cohen C, Levin G, Patel KV, de Boer $\mathbb{H}$, Seliger S, Ruzinski J, Himmelfarb J, Kestenbaum B. A prospective study of frailty in nephrology-referred patients with CKD. Am J Kidney Dis. 2012; 60(6):912-21.

10. Fried LF, Lee JS, Shlipak M, Chertow GM, Green C, Ding J, Harris T, Newman AB. Chronic kidney disease and functional limitation in older people: health, aging and body composition study. J Am Geriatr Soc. 2006;54(5):750-6.

11. Kurella M, Chertow GM, Fried LF, Cummings SR, Harris T, Simonsick E, Satterfield S, Ayonayon H, Yaffe K. Chronic kidney disease and cognitive impairment in the elderly: the health, aging, and body composition study. J Am Soc Nephrol. 2005;16(7):2127-33.

12. Deva R, Alias MA, Colville D, Tow FK, Ooi QL, Chew S, Mohamad N, Hutchinson A, Koukouras I, Power DA, et al. Vision-threatening retinal abnormalities in chronic kidney disease stages 3 to 5. Clin J Am Soc Nephrol. 2011;6(8):1866-71.

13. Duenhas MR, Draibe SA, Avesani CM, Sesso R, Cuppari L. Influence of renal function on spontaneous dietary intake and on nutritional status of chronic renal insufficiency patients. Eur J Clin Nutr. 2003;57(11):1473-8.

14. Foley RN, Wang C, Ishani A, Collins AJ, Murray AM. Kidney function and sarcopenia in the United States general population: NHANES III. Am J Nephrol. 2007;27(3):279-86.

15. Picariello F, Moss-Morris R, Macdougall IC, Chilcot AJ. The role of psychological factors in fatigue among end-stage kidney disease patients: a critical review. Clin Kidney J. 2017;10(1):79-88.

16. Ju A, Unruh ML, Davison SN, Dapueto J, Dew MA, Fluck R, Germain M, Jassal SV, Obrador G, O'Donoghue D, et al. Patient-reported outcome measures for fatigue in patients on hemodialysis: a systematic review. Am J Kidney Dis. 2018;71(3):327-43.

17. Rosansky SJ. Renal function trajectory is more important than chronic kidney disease stage for managing patients with chronic kidney disease. Am J Nephrol. 2012;36(1):1-10.

18. Kalender B, Ozdemir AC, Dervisoglu E, Ozdemir O. Quality of life in chronic kidney disease: effects of treatment modality, depression, malnutrition and inflammation. Int J Clin Pract. 2007;61(4):569-76.

19. Perlman RL, Finkelstein FO, Liu L, Roys E, Kiser M, Eisele G, Burrows-Hudson S, Messana JM, Levin N, Rajagopalan S, et al. Quality of life in chronic kidney disease (CKD): a cross-sectional analysis in the renal Research institute-CKD study. Am J Kidney Dis. 2005;45(4):658-66.

20. Yapa HE, Purtell L, Chambers S, Bonner A. The relationship between chronic kidney disease, symptoms and health-related quality of life: a systematic review. J Ren Care. 2020:46.2:74-84.

21. Stevens PE, O'Donoghue DJ, de Lusignan S, Van Vlymen J, Klebe B, Middleton R, Hague N, New J, Farmer CK. Chronic kidney disease management in the United Kingdom: NEOERICA project results. Kidney Int. 2007:72(1):92-9

22. Cirillo $M$, Laurenzi $M$, Mancini $M$, Zanchetti A, Lombardi C, De Santo NG. Low glomerular filtration in the population: prevalence, associated disorders, and awareness. Kidney Int. 2006;70(4):800-6.

23. Corsonello A, Tap L, Roller-Wirnsberger R, Wirnsberger G, Zoccali C, Kostka T, Guligowska A, Mattace-Raso F, Gil P, Fuentes LG, et al. Design and methodology of the screening for CKD among older patients across Europe (SCOPE) study: a multicenter cohort observational study. BMC Nephrol. 2018;19(1):260

24. Rabin R, de Charro F. EQ-5D: a measure of health status from the EuroQol group. Ann Med. 2001;33(5):337-43.

25. Balestroni $G$, Bertolotti $G$. EuroQol-5D (EQ-5D): an instrument for measuring quality of life. Monaldi Arch Chest Dis. 2012;78(3):155-9.
26. Oemar M, Oppe M. EQ-5D-3L User Guide. EuroQol Group. 2013. http://www eurogol.org/fileadmin/user_upload/Documenten/PDF/Folders_Flyers/EQ-5 D-3L_UserGuide_2013_v5.0_October_2013.pdf.

27. Brazier J, Connell J, Papaioannou D, Mukuria C, Mulhern B, Peasgood T, Jones ML, Paisley S, O'Cathain A, Barkham M, et al. A systematic review, psychometric analysis and qualitative assessment of generic preferencebased measures of health in mental health populations and the estimation of mapping functions from widely used specific measures. Health Technol Assess. 2014;18(34) vii-viii, xiii-xxv:1-188.

28. Yang $Y$, Brazier J, Longworth L. EQ-5D in skin conditions: an assessment of validity and responsiveness. Eur J Health Econ. 2015;16(9):927-39.

29. Davies N, Gibbons E, Mackintosh A, Fitzpatrick R. A structured review of patient-reported outcome measures for women with breast cancer. In: Patient-reported Outcome Measurement Group, Department of Public Health, University of Oxford; 2009. http://phi.uhce.ox.ac.uk/pdf/ CancerReviews/PROMs_Oxford_BreastCancer_012011.pdf.

30. Pickard AS, Wilke C, Jung E, Patel S, Stavem K, Lee TA. Use of a preferencebased measure of health (EQ-5D) in COPD and asthma. Respir Med. 2008; 102(4):519-36.

31. Schaeffner ES, Ebert N, Delanaye P, Frei U, Gaedeke J, Jakob O, Kuhlmann MK, Schuchardt M, Tolle M, Ziebig R, et al. Two novel equations to estimate kidney function in persons aged 70 years or older. Ann Intern Med. 2012; 157(7):471-81.

32. Katz $\mathrm{S}$, Ford $A B$, Moskowitz RW, Jackson BA, Jaffe MW. Studies of illness in the aged. The index of Adl: a standardized measure of biological and psychosocial function. JAMA. 1963;185:914-9.

33. Lawton MP, Brody EM. Assessment of older people: self-maintaining and instrumental activities of daily living. Gerontologist. 1969;9(3):179-86.

34. Folstein MF, Folstein SE, McHugh PR. "Mini-mental state". A practical method for grading the cognitive state of patients for the clinician. J Psychiatr Res. 1975;12(3):189-98.

35. Lesher EL, Berryhill JS. Validation of the geriatric depression scale--short form among inpatients. J Clin Psychol. 1994;50(2):256-60.

36. Conwell Y, Forbes NT, Cox C, Caine ED. Validation of a measure of physica illness burden at autopsy: the cumulative illness rating scale. J Am Geriatr Soc. 1993;41(1):38-41.

37. Rosenberg MT, Staskin DR, Kaplan SA, MacDiarmid SA, Newman DK, Ohl DA. A practical guide to the evaluation and treatment of male lower urinary tract symptoms in the primary care setting. Int I Clin Pract. 2007;61 (9):1535-46.

38. Morley JE, Abbatecola AM, Argiles JM, Baracos V, Bauer J, Bhasin S, Cederholm T, Coats AJ, Cummings SR, Evans WJ, et al. Sarcopenia with limited mobility: an international consensus. J Am Med Dir Assoc. 2011; 12(6):403-9.

39. Guralnik JM, Fried LP, Salive ME. Disability as a public health outcome in the aging population. Annu Rev Public Health. 1996;17(1):25-46.

40. Almutary H, Bonner A, Douglas C. Symptom burden in chronic kidney disease: a review of recent literature. J Ren Care. 2013:39(3):140-50.

41. Murtagh FE, Addington-Hall J, Higginson IJ. The prevalence of symptoms in end-stage renal disease: a systematic review. Adv Chronic Kidney Dis. 2007; 14(1):82-99.

42. Canney M, Sexton E, Tobin K, Kenny RA, Little MA, O'Seaghdha CM. The relationship between kidney function and quality of life among communitydwelling adults varies by age and filtration marker. Clin Kidney J. 2018;11(2): 259-64.

43. O'Hare AM, Rodriguez RA, Bowling CB. Caring for patients with kidney disease: shifting the paradigm from evidence-based medicine to patientcentered care. Nephrol Dial Transplant. 2016;31(3):368-75.

44. Lattanzio F, Corsonello A, Montesanto A, Abbatecola AM, Lofaro D, Passarino G, Fusco S, Corica F, Pedone C, Maggio M, et al. Disentangling the impact of chronic kidney disease, Anemia, and mobility limitation on mortality in older patients discharged from hospital. J Gerontol A Biol Sci Med Sci. 2015;70(9):1120-7.

45. Trombetti A, Reid KF, Hars M, Herrmann FR, Pasha E, Phillips EM, Fielding RA. Age-associated declines in muscle mass, strength, power, and physical performance: impact on fear of falling and quality of life. Osteoporos Int. 2016;27(2):463-71.

46. Perera S, Mody SH, Woodman RC, Studenski SA. Meaningful change and responsiveness in common physical performance measures in older adults. J Am Geriatr Soc. 2006;54(5):743-9.

47. Hargrove N, Tays Q, Storsley L, Komenda P, Rigatto C, Ferguson T, Tangri N, Bohm C. Effect of an exercise rehabilitation program on physical function 
over 1 year in chronic kidney disease: an observational study. Clin Kidney J. 2020;13(1):95-104.

48. Taryana AA, Krishnasamy R, Bohm C, Palmer SC, Wiebe N, Boudville N, MacRae J, Coombes JS, Hawley C, Isbel N, et al. Physical activity for people with chronic kidney disease: an international survey of nephrologist practice patterns and research priorities. BMJ Open. 2019;9(12):e032322.

49. Kasiske BL, Wheeler DC. KDIGO clinical practice guideline for the evaluation and Management of Chronic Kidney Disease Foreword. Kidney Int Suppl. 2013;3(1):2-2

50. Incalzi RA, Corsonello A, Pedone C, Battaglia S, Paglino G, Bellia V, COPD EC. Chronic renal failure a neglected comorbidity of COPD. Chest. 2010;137(4): 831-7.

51. Mingardi G, Cornalba L, Cortinovis E, Ruggiata R, Mosconi P, Apolone G. Health-related quality of life in dialysis patients. A report from an Italian study using the SF-36 health survey. DIA-QOL group. Nephrol Dial Transplant. 1999;14(6):1503-10.

52. Merkus MP, Jager KJ, Dekker FW, de Haan RJ, Boeschoten EW, Krediet RT. Physical symptoms and quality of life in patients on chronic dialysis: results of the Netherlands cooperative study on adequacy of Dialysis (NECOSAD). Nephrol Dial Transplant. 1999;14(5):1163-70

53. Baiardi F, Degli Esposti E, Cocchi R, Fabbri A, Sturani A, Valpiani G, Fusarol M. Effects of clinical and individual variables on quality of life in chronic renal failure patients. J Nephrol. 2002;15(1):61-7.

54. Jing X, Chen J, Dong Y, Han D, Zhao H, Wang X, Gao F, Li C, Cui Z, Liu Y, et al. Related factors of quality of life of type 2 diabetes patients: a systematic review and meta-analysis. Health Qual Life Outcomes. 2018;16(1): 189.

55. Wang Q, Liu X, Zhu M, Pang H, Kang L, Zeng P, Ge N, Qu X, Chen W, Hong $X$. Factors associated with health-related quality of life in communitydwelling elderly people in China. Geriatr Gerontol Int. 2020;20(5):422-9.

56. Montiel-Luque A, Nunez-Montenegro AJ, Martin-Aurioles E, Canca-Sanchez JC, Toro-Toro MC, Gonzalez-Correa JA, Polipresact Research G. Medicationrelated factors associated with health-related quality of life in patients older than 65 years with polypharmacy. PLoS One. 2017;12(2):e0171320.

\section{Publisher's Note}

Springer Nature remains neutral with regard to jurisdictional claims in published maps and institutional affiliations.

Ready to submit your research? Choose BMC and benefit from:

- fast, convenient online submission

- thorough peer review by experienced researchers in your field

- rapid publication on acceptance

- support for research data, including large and complex data types

- gold Open Access which fosters wider collaboration and increased citations

- maximum visibility for your research: over $100 \mathrm{M}$ website views per year

At $\mathrm{BMC}$, research is always in progress.

Learn more biomedcentral.com/submissions 